\title{
Atomistic simulations of the mechanical behavior of fivefold twinned nanowires
}

\author{
Ajing Cao* and Yueguang $\mathrm{Wei}^{\dagger}$ \\ State Key Laboratory of Nonlinear Mechanics, Institute of Mechanics, Chinese Academy of Sciences, \\ Beijing 100080, People's Republic of China
}

(Received 31 July 2006; revised manuscript received 18 November 2006; published 13 December 2006)

\begin{abstract}
Atomistic simulations are used to investigate the mechanical behavior of metal nanowire with fivefold twinned structure. The twinned nanowires were reported in recent experiments [B. Wu et al., Nano Lett. 6, 468 (2006)]. In the present paper, we find that the yield strength of the fivefold twinned $\mathrm{Cu}$ nanowire is $1.3 \mathrm{GPa}$ higher than that of the face-centered-cubic (fcc) $\langle 110\rangle$ single crystalline $\mathrm{Cu}$ nanowire without fivefold twinned structure, and the microstructure-hardened mechanism is primarily due to the twinned boundaries which act as the barriers for the dislocation emission and propagation. However, we also find that the fivefold twinned $\mathrm{Cu}$ nanowire has lower ductility than that of fcc $\langle 110\rangle$ single crystalline $\mathrm{Cu}$ nanowire without the twinned structure, and this is mainly attributed to the scarcity and low mobility of dislocations. In addition, in our simulations the effect of preexisting stacking faults and dislocations on strength of the fivefold twinned nanowires is investigated.
\end{abstract}

DOI: $10.1103 /$ PhysRevB.74.214108

PACS number(s): 61.46. $-\mathrm{w}, 62.25 .+\mathrm{g}$

\section{INTRODUCTION}

Due to the unique properties in mechanical, electronic, optical, and magnetic aspects, the nanostructure materials have attracted a great deal of interest in the past few decades. ${ }^{1-7}$ Metal nanowires are of technological importance because of their potential applications in miniaturizations of the systems concerned with the electrical, optical, thermal, and mechanical aspects. Although a variety of metal nanowires (such as the gold, copper, and silver nanowires) have been synthesized, the mechanical properties of freestanding nanostructured materials at a single-object level still need to be investigated.

Nanostructure design and control have attracted a great deal of attention. Wu et al. ${ }^{8}$ pointed out that in nanowire systems nanostructure design was a particularly effective means for controlling the mechanical properties. Silver nanowires with a unique fivefold twinned structure were synthesized and their mechanical properties were examined by means of three-point bending test method. ${ }^{8,9}$ The results showed that the unique nanostructure played a significant role in controlling the strength and toughness of metal nanowires. However, the effects of the nanostructure on the mechanical properties are not well understood only from the previous experiments, and the effective atomic-level mechanisms still need to be explored.

In the present research, mechanical behavior of metal nanowires with fivefold twinned structure recently observed in experiments will be investigated by using atomistic simulations. Special attention is focused on setting up the correlation between the unique nanostructure and mechanical properties. In addition, effort is paid to explore detail strengthening mechanisms of the nanowires at the atomic level in order to obtain a fundamental understanding of mechanical behaviors for the nanostructured materials. Furthermore, the elastic, plastic, and failure behavior of the nanowires with the unique structure are simulated and analyzed.

\section{SIMULATION METHODS}

In this work, the purely semiempirical potential named embedded-atom method (EAM) (Refs. 10 and 11) is adopted for material $\mathrm{Cu}$. The EAM method is based upon the recognition that cohesive energy of a metal is governed not only by the pairwise potential of nearest-neighbor atoms, but also by the embedding energy related to the "electron sea" in which the atom is embedded. The cohesive energy is described by

$$
U_{\text {total }}=\sum_{i} F_{i}\left(\rho_{i}\right)+\frac{1}{2} \sum_{j \neq i} \Phi_{i j}\left(r_{i j}\right),
$$

where $U_{\text {total }}$ is the total internal energy, $F_{i}$ is the embedding energy of the $i$ th atom, $\rho_{i}$ is the electron density at atom $i$, and $r_{i j}$ is the distance between atom $i$ and atom $j . \Phi_{i j}$ is the pairwise electrostatic interaction energy. The parameters in the EAM potential are fit to the equilibrium lattice constant, elastic constants, cohesive energy, surface energy, stacking fault energy, sublimation energy, as well as vacancy formation energy. ${ }^{11}$ Table I shows the parameter values for material $\mathrm{Cu}$ adopted in the present work.

The Virial stress for the atom system is defined ${ }^{12,13}$ as

$$
\Pi^{\alpha \beta}=\frac{1}{\Omega}\left\{-\sum_{i} m_{i} \nu_{i}^{\alpha} \nu_{i}^{\beta}+\frac{1}{2} \sum_{i} \sum_{j \neq i}\left(-\frac{1}{r_{i j}} \frac{\partial U_{\text {total }}}{\partial r_{i j}}\right) r_{i j}^{\alpha} r_{i j}^{\beta}\right\},
$$

where $\Omega$ is the volume of the system, $m_{i}$ and $\nu_{i}$ are the mass and velocity of atom $i$, and the indices $\alpha$ and $\beta$ denote the Cartesian components. $r_{i j}^{\alpha}$ is the projection of the interatomic distance vector $\mathbf{r}_{\mathbf{i j}}=\mathbf{r}_{\mathbf{i}}-\mathbf{r}_{\mathbf{j}}$ along coordinate $\alpha$, and $r_{i j}=\left|\mathbf{r}_{\mathbf{i j}}\right|$.

TABLE I. Material properties associated with the EAM potential for $\mathrm{Cu}$ tabulated by Foiles et al. (Ref. 11).

\begin{tabular}{lcccccc}
\hline \hline & $\begin{array}{c}\text { Lattice } \\
\text { constant } \\
(\AA)\end{array}$ & $\begin{array}{c}\mathrm{C} 11 \\
(\mathrm{GPa})\end{array}$ & $\begin{array}{c}\mathrm{C} 12 \\
(\mathrm{GPa})\end{array}$ & $\begin{array}{c}\mathrm{C} 44 \\
(\mathrm{GPa})\end{array}$ & $\begin{array}{c}E_{\text {Sublimation }} \\
(\mathrm{ev})\end{array}$ & $\begin{array}{c}E_{\text {vacancy }}^{f} \\
(\mathrm{ev})\end{array}$ \\
\hline $\begin{array}{l}\text { EAM } \\
\text { Potential }\end{array}$ & 3.615 & 167 & 124 & 76 & 3.54 & 1.28 \\
\hline \hline
\end{tabular}


The mean stress of the whole system (Virial stress, $\Pi^{\alpha \beta}$ ) described by Eq. (2) is same as the Cauchy stress. We also adopt the atomic level stress associated with atom $i$ which is obtained by decomposing the Virial stress onto every atom,

$$
\Pi^{\alpha \beta}=\frac{1}{\Omega} \sum_{i} \omega_{i} \pi_{i}^{\alpha \beta}
$$

with

$$
\pi_{i}^{\alpha \beta}=\frac{1}{\omega_{i}}\left\{-m_{i} \nu_{i}^{\alpha} \nu_{i}^{\beta}+\frac{1}{2} \sum_{j \neq i}\left(-\frac{1}{r_{i j}} \frac{\partial U_{\text {total }}}{\partial r_{i j}}\right) r_{i j}^{\alpha} r_{i j}^{\beta}\right\}
$$

and $\sum \omega_{i}=\Omega$. Obviously, Eq. (4) can be taken as the definition of an atomic level stress associated with atom $i$, where $\omega_{i}$ is the effective volume of the atom $i .{ }^{14}$ Strain is defined as $\varepsilon$ $=\left(l-l_{0}\right) / l_{0}$, where $l$ is the current wire length and $l_{0}$ is the initial length.

In order to discern defects in the nanowires, colors are assigned to the atoms according to a local crystallinity classification visualized by common neighbor analysis (CNA), ${ }^{15}$ which permits the distinction between atoms in a local hexagonal-close-packed (hcp) environment and those in a face-centered-cubic (fcc) environment. Grey (gray) stands for fcc atoms, red (dark) for hcp atoms, green (dark gray) for the other twelve coordinated atoms and blue for the nontwelve coordinated atoms. A single line of hcp atoms represents a twin boundary, two adjacent hep lines stand for an intrinsic stacking fault, and two hep lines with a fcc line stand for an extrinsic stacking fault between them. The other twelve coordinated atoms and non-twelve coordinated atoms appear in the free surface region and in the core of dislocations.

Here a $\mathrm{Cu}$ nanowire with novel uniform fivefold twinned structure along the entire wire length (hereafter denoted as the fivefold twinned wire) with square cross section is considered. First, the initial state is constructed by rotating $\Sigma 3$ coherent twins about $\langle 110\rangle$ axis according to coincidence site lattice. ${ }^{16}$ Second, a cut is made to form a nearly square cross section with $8 \mathrm{~nm} \times 8 \mathrm{~nm}$, and the cut side surfaces of the wire consist of the planes $\{111\},\{112\},\{001\}$, and also $\{551\}$. Third, the conjugate gradient method is used to minimize the whole wire energy to obtain a more stable configuration, and a thermal equilibrium processing to $300 \mathrm{~K}$ for $20 \mathrm{ps}$ is performed by using a Nosé-Hoover thermostat ${ }^{17,18}$ while keeping the length of the wires constant. The equilibrated configuration is shown in Fig. 1.

For comparison, another case of simulation on fcc single crystalline $\langle 110\rangle \mathrm{Cu}$ nanowire which has the same wire length direction as the fivefold twinned nanowire is performed. To be possibly close to the side surface of the fivefold twinned nanowire, the $\langle 110\rangle$ single crystalline $\mathrm{Cu}$ nanowire is chosen to have $\langle 110\rangle$ longitudinal orientation with two $\{112\}$ side surfaces, and with two $\{111\}$ side surfaces which are created with initial atomic positions corresponding to those of the bulk fcc $\mathrm{Cu}$ lattice. (Hereafter denoted as $\langle 110\rangle$ single crystalline wire or configuration). Both nanowires have the same axial length $(30 \mathrm{~nm})$ and lateral side length $(8 \mathrm{~nm})$. It is worth emphasizing again that the key

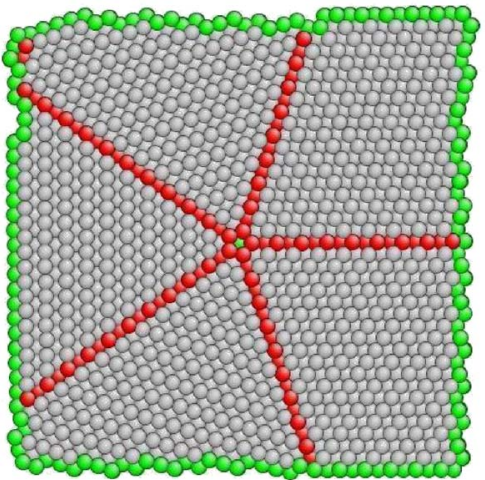

(a) (b)

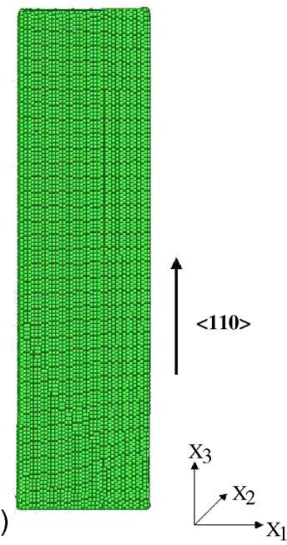

FIG. 1. (Color online) Equilibrated fivefold twinned $\mathrm{Cu}$ nanowire. (a) Cross-sectional view; (b) side view with axis orientation of $\langle 110\rangle$. Atoms are colored according to CNA.

difference between two nanowires considered here is that the first wire contains the novel twin structures and the second one does not. Free boundary conditions are used in all three dimensions.

To explore the mechanical behavior of metal nanowires, uniaxial tensile loading is exerted under quasistatic conditions. ${ }^{19-26}$ To accelerate simulation efficiency, in the first eight loading steps, all the atoms are first displaced in a prescribed uniform strain increment of $0.5 \%$ in the length direction, and then in the remaining steps, $0.2 \%$ strain increment is applied for each step. The wires are then relaxed under keeping the constant temperature $300 \mathrm{~K}$ for $25 \mathrm{ps}$ at their terminals to obtain a macroscopic equilibrium configuration. The equilibrated process usually takes less than $15 \mathrm{ps,}$ and the mean stress over the last $5 \mathrm{ps}$ of the relaxation period is taken as the stress of the nanowire. The stresses are calculated using the Virial theorem, which is equivalent to the Cauchy stress in the mean sense. ${ }^{12}$

\section{RESULTS AND DISCUSSION}

Figure 2 shows the tensile stress strain curves for the two kinds of nanowires. Clearly, the curves for two nanowires essentially coincide with each other during the elastic deformation. The Young's modulus calculated from the stress strain curve is about $130 \mathrm{GPa}$ for the $\langle 110\rangle$ type crystalline directions. After the elastic deformation, the stress strain curves display precipitous drops, which implies that the plastic yielding takes place in the nanowires. The yielding stress of the fivefold twinned $\mathrm{Cu}$ nanowire is $4.8 \mathrm{GPa}$, while the yielding stress of $\langle 110\rangle \mathrm{Cu}$ wire is $3.5 \mathrm{GPa}$. There is an obvious strength increase, $1.3 \mathrm{GPa}$, a $30 \%$ increase of the strength, which is a considerably high strength increase in the nanowire systems. However, the ductility of the nanowire with the fivefold twinned structure is much lower than that of the $\langle 110\rangle \mathrm{Cu}$ nanowire. This feature will be discussed later.

Usually, atomistic simulation results critically depend on the adopted potential. For investigating the result difference due to different potential used, here we also perform the atomistic simulations on the above two wires by adopting a 


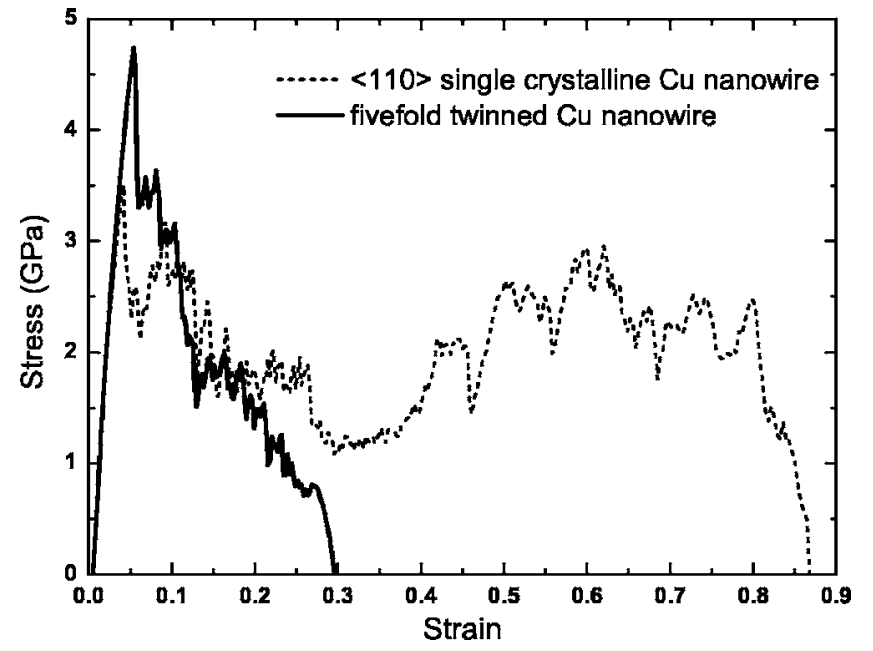

FIG. 2. Tensile stress-strain curves for the fivefold twinned $\mathrm{Cu}$ nanowire and for the $\langle 110\rangle$ single crystalline $\mathrm{Cu}$ nanowire.

version of the EAM potential developed by Mishin et al. ${ }^{27}$ The initial yielding stress-strain responses based on both Mishin EAM potential and Foiles potential are plotted in Fig. 3. The strength difference between fivefold twinned $\mathrm{Cu}$ nanowire and $\langle 110\rangle \mathrm{Cu}$ nanowire simulated by using two potentials is not big. Essentially the deformation mechanisms of the nanowires are the same for using the above two potentials. In the following section, we shall concentrate our attention on the resulting discussions for using the Foiles potential case.

Due to the surface stress, ${ }^{19-21}$ the initial free-standing nanowire is not in equilibrium and it will undergo contraction in the length direction. The surface stress (tensile stress) induces an "intrinsic" compressive stress in the core part of the nanowire. To evaluate the surface stress contribution to the yielding strength, we calculate the distribution of atomic level stress on the cross section of the two nanowires. Because uniaxial loading is applied to the nanowires, only $\Pi^{33}$ is nonzero and is equal to the external force divided by the

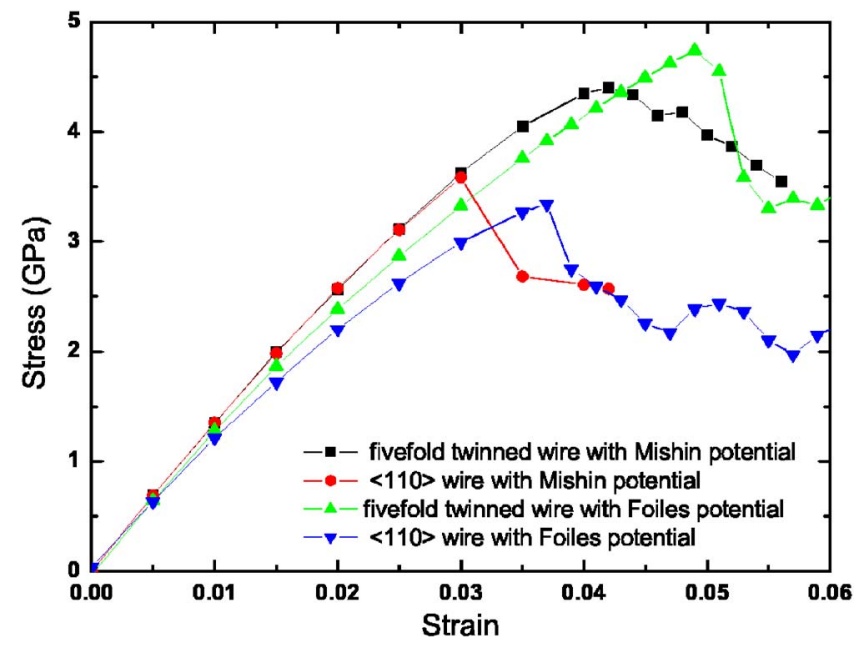

FIG. 3. (Color online) Tensile stress-strain curves for the fivefold twinned $\mathrm{Cu}$ nanowire and the $\langle 110\rangle$ single crystalline $\mathrm{Cu}$ nanowire using both Foiles EAM potential (Ref. 11) and Mishin EAM potential (Ref. 27).

cross-sectional area. The atomic level stress (normal stress component, $\pi^{33}$ ) for the fivefold twinned $\mathrm{Cu}$ nanowires is shown in Fig. 4(a). The stress becomes heterogeneous in the interior after relaxation for the preexisting twin structure. Near and far away twin boundaries the stress distributions are in quite a different way. Especially, the stress on the center of the wire is in the largest compressive state and it changes with a gradient to the tensile stress state (at the surface). This indicates that the internal twin structures cause the change of the internal stress distribution. Magnitude of surface tensile stress depends on the surface orientation. From Fig. 4, on $\{111\}$ planes there is larger surface stress than on $\{112\}$ planes. However, for the $\langle 110\rangle \mathrm{Cu}$ nanowire without twinned structures, the stress is quite uniform in the interior cross section and it has a mean value, $-0.6 \mathrm{GPa}$, and the magnitude of stress is lower in the $\langle 110\rangle \mathrm{Cu}$ nanowire than that in the fivefold twinned $\mathrm{Cu}$ wire.

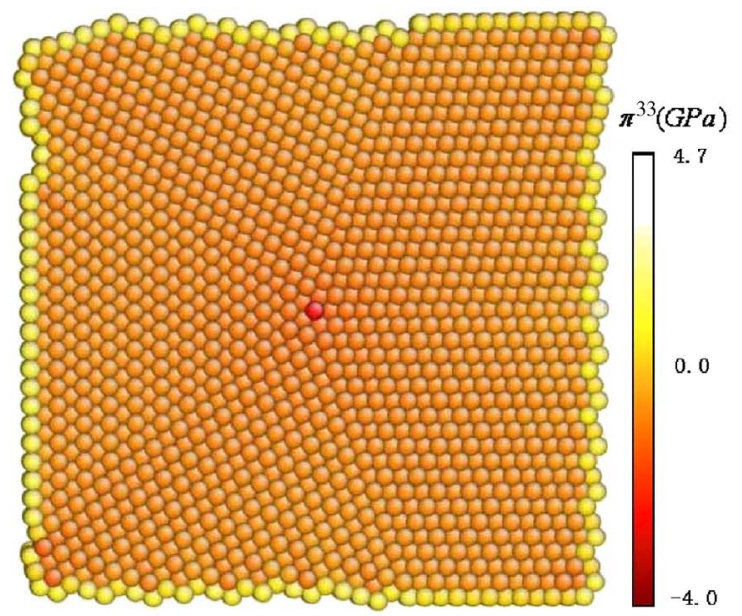

(a)

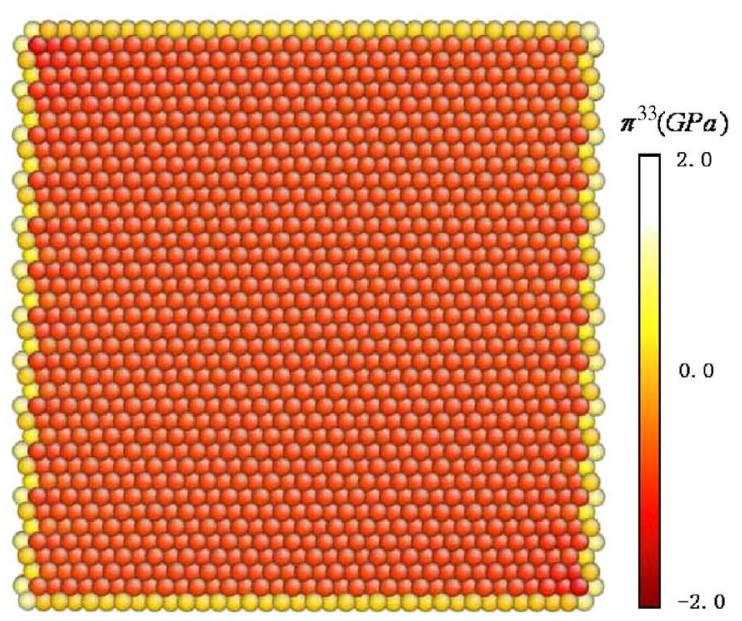

(b)

FIG. 4. (Color online) Distribution of atomic level stress, $\pi^{33}$, on the cross section of the (a) relaxed fivefold twinned Cu nanowire and (b) relaxed $\langle 110\rangle$ single crystalline $\mathrm{Cu}$ nanowire with two $\{112\}$ and two $\{111\}$ side surfaces. 
(a)

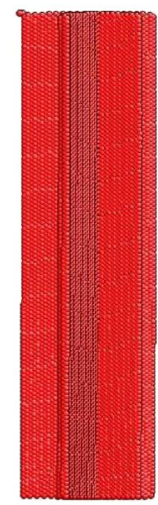

(b)

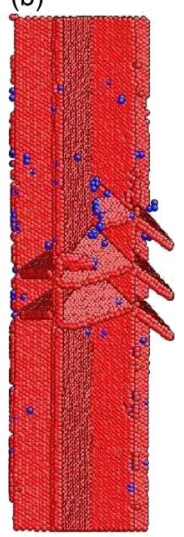

(c)

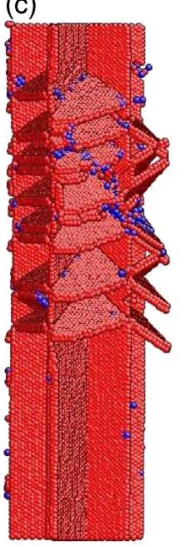

(d)

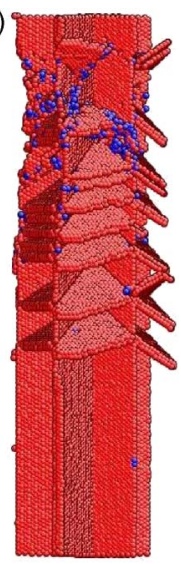

FIG. 5. (Color online) Tensile yielding of fivefold twinned $\mathrm{Cu}$ nanowires with $\{111\}\langle 112\rangle$ dislocations pile-up at twin boundaries. (a) Initial configuration of wire with fivefold twin structure, (b) initial yield occurring by dislocation nucleation and intersection with twin boundaries, (c) partial dislocations and stacking faults left in the wire interior along the wire axis, (d) necking set in at strain 0.12. Atoms are colored according to CNA. For clarity perfect fcc atoms and surface atoms are removed to visualize interior defects.

Figure 5 shows the detail of yielding deformation of fivefold twinned $\mathrm{Cu}$ wire. As can be seen, first yielding occurs via $\frac{1}{6}\langle 112\rangle$ partial dislocation nucleation at surface edge, and then the dislocations glide to the center of the wire in the $\{111\}$ slip planes while further movement is restricted by the fivefold twin boundaries. The twin boundaries take the action as a barrier for dislocation gliding in the slip planes, and then serials of stacking faults and dislocations are left along the wire length.

Although one-dimensional structure of the nanowires is considered, the stress state in the wires is essentially a threedimensional stress state due to the interior microstructure. The $\{111\}$ twin boundaries divide the nanowires into five nanograins (see Fig. 6). In this perspective, the grain size is refined and the much higher shear stress is needed to nucleate a dislocation than that in single-crystalline nanowire.

However, like nanocrystalline materials, these novel twinned nanowires sacrifice the elongation ductility. This is consistent with experimental observation. ${ }^{8}$ Figure 7 displays (a)

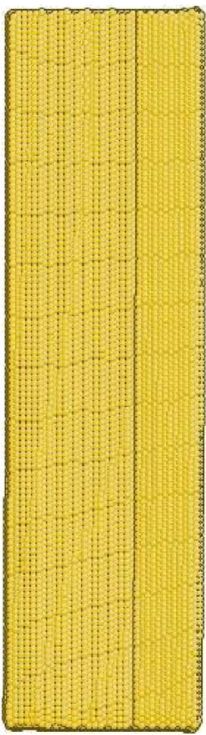

(b)

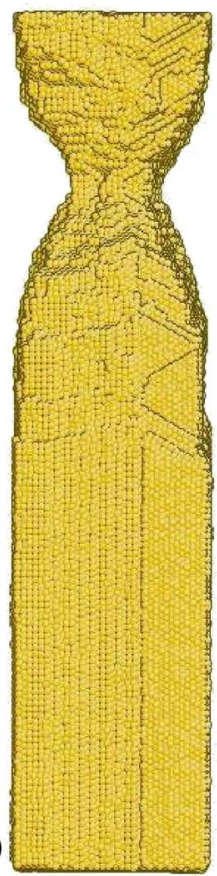

(c)
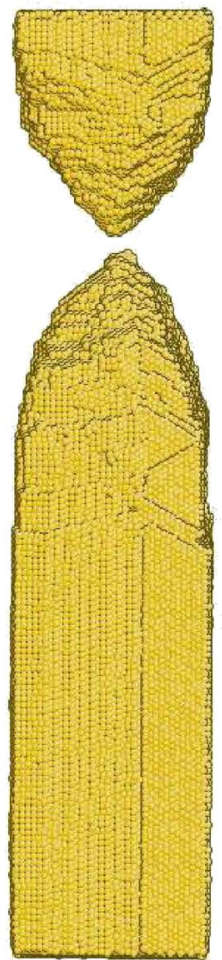

FIG. 7. (Color online) Brittle tensile failure of fivefold twinned $\mathrm{Cu}$ nanowire. (a) Initial equilibrated configuration, (b) strain at 0.17 , (c) ultimate fracture strain at 0.29 .

the failure snapshots of fivefold twinned wire. The twin boundaries restrict dislocation motion, and plastic deformation is limited since stress concentration cannot be released in the wire. These factors cause necking instability and failure. As deformation increases, necking sets in and leads to failure. The ultimate fracture strain is approximately 0.29, which is significantly lower than that of wire without the twinned nanostructure, 0.86. (See Fig. 2.)

In contrast, the major tensile deformation mechanism of $\langle 110\rangle \mathrm{Cu}$ wire is via partial dislocation slip in $\{111\}$ planes and twin boundary propagation along the wire axis. Recently, molecular dynamics (MD) simulations have shown that both shape memory and pseudoelasticity are observed in a class of fcc nanowires with lateral sizes about $5 \mathrm{~nm}$ or smaller when

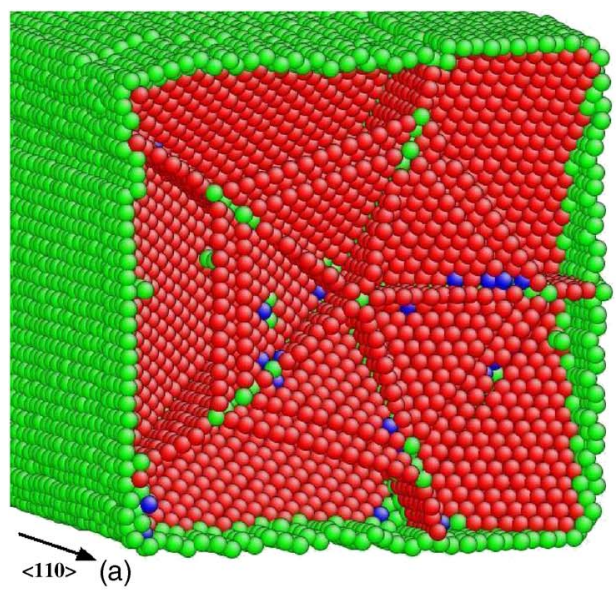

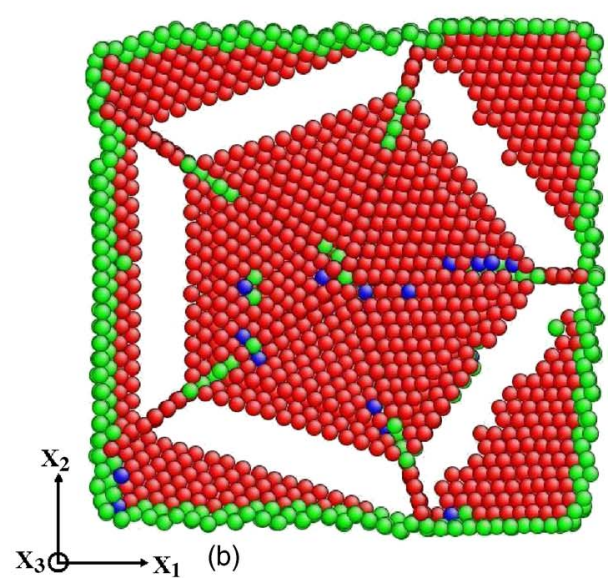

FIG. 6. (Color online) Snapshot of Shockley partial dislocations and stacking faults intercepted with twin boundaries. (a) View of stacking fault intercepted by view plane with pentagonal geometry, (b) view along wire length with only eight (110) plane atoms. Atoms are colored according to CNA. To facilitate visualizing defects, fcc atoms are not shown in the wire. 


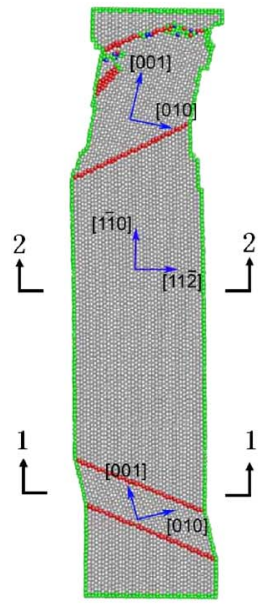

(a)

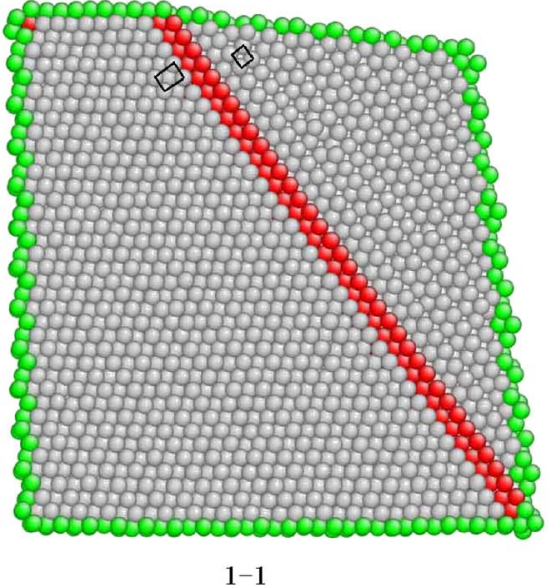

(b)

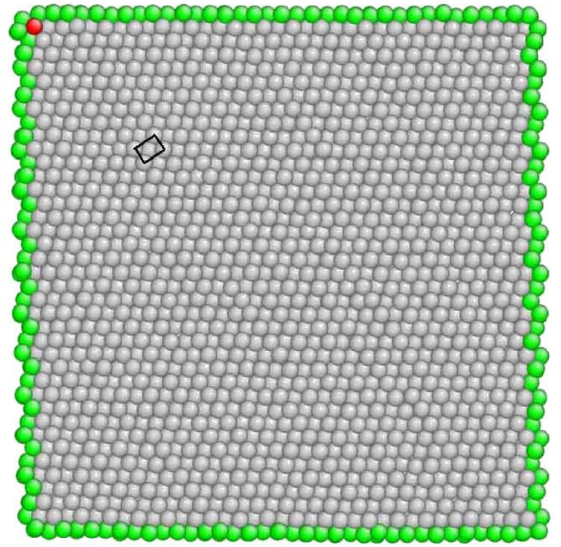

$2-2$

(c)

FIG. 8. (Color online) First stage of lattice orientation of the $\langle 110\rangle$ single-crystalline $\mathrm{Cu}$ nanowire at a strain of 0.2 . (a) Sectional view along the wire axis and [112] of the cross section, the different lattice orientation is separated by twin boundaries and the wire axis direction is also shown. (b) Cross-section 1-1 shows the transition region containing both $\langle 100\rangle$ and $\langle 110\rangle$ lattice configuration. (c) Cross-section 2-2 shows the initial $\langle 110\rangle$ lattice configuration. Atoms are colored according to CNA.

loading is exerted along the $\langle 110\rangle$ with $\{111\}$ side surface configuration. ${ }^{23,24,26}$ In fact, under the uniaxial tensile loading at room temperature, the $\langle 110\rangle$ wire with $\{111\}$ and $\{112\}$ side surfaces also displays the lattice reorientation within the fcc crystalline structure. The deformation process consists of two major steps: (1) $\langle 110\rangle$ wires reorient to $\langle 100\rangle$ wires with $\{100\}$ and $\{112\}$ side surfaces; (2) the newly formed $\langle 100\rangle$ wires further reorient to $\langle 112\rangle$ wires with $\{111\}$ side surfaces. The first lattice reorientation is based on the mechanical twinning of fcc lattice, which is the result of consecutive nucleation, gliding, and annihilation of $\frac{1}{6}\langle 112\rangle$ Shockley partial dislocations, essentially the same as the tension of $\langle 110\rangle /\{111\}$ wire to $\langle 100\rangle /\{100\}$ configuration. The twin boundaries nucleate from both terminals of the wires and propagate toward each other, which leads to the reorientation to the $\langle 001\rangle$ wire with $\{100\}$ and $\{112\}$ side surfaces, as illustrated in Fig. 8. The full reorientation is completed at strain 0.47 . The initial square cross section is transformed into rhombic cross section with both $\{100\}$ and $\{112\}$ side surfaces. This is the first stage of the transform process, which was also found in recent work. ${ }^{23,24,26}$ In the second stage, twin boundaries nucleate and propagate, which leads to the lattice reorientation from $\langle 100\rangle$ wire to $\langle 112\rangle$ wire. The transformed wire configuration is shown in Fig.9. The initial twin boundaries are formed in the upper wire and propagate consecutively downward by an interplanar distance between two neighboring $\{111\}$ planes. The twin boundary propagation is stopped by the necking and failure of the wire at 0.87 of the strain value, so the lattice reorientation is not completed in the whole wires. The failed wire has both $\langle 100\rangle$ and $\langle 112\rangle$ axis directions, which is separated by twin boundaries. From the above observation and analyses, we think that the surface stresses play an important role in size-limiting the reorientation from $\langle 110\rangle$ wire via $\langle 100\rangle$ wire to $\langle 112\rangle$ wire.

It is worth noting that the tensile ductility of the $\langle 110\rangle$ wire is much higher than that simulated by Liang et al.; ${ }^{24}$ this is because the different lattice reorientation stage is involved. There are different side surfaces in the present simulation (two $\{112\}$ side surfaces and two $\{110\}$ side surfaces) from that in the work of Liang et al. (four $\{111\}$ side surfaces). The structures of fully reconstructed nanowire here are similar to those of experimental observations, in which an initially rod-shaped gold nanowire [110] was stretched and the apex tip with a [111] axis close to the stretching direction was observed. ${ }^{28}$

Both dislocation slip and twin boundary propagation together cause the wire necking and final failure. The ultimate fracture strain can reach about 0.86 , which is significantly higher than that for bulk counterparts. The snapshot of the failure is shown in Fig. 10.

Furthermore, we also made a comparison between the perfect fivefold twinned $\mathrm{Cu}$ wires and the same wire with a stacking fault (SF) within it (Fig. 11). Figure 12 shows the tensile stress strain curves of the two wires. The wire with a SF shows a decrease in strength after peak stress occurs. But the SF has a little influence on the yielding strength. In contrast, the yielding stress was considerably affected by the preexisting defects in the $\langle 110\rangle$ nanowires. ${ }^{20}$

The hardened mechanism in metal nanowires is different from that in bulk coarse-grain materials. Conventional hardening mechanisms, such as cold-work hardening and precipitation hardening, would not take effect in describing the nanostructures. Nanostructure-controlling mechanisms are widely believed to play a significant role in tailoring mechanical properties, such as the nanowires in the present research.

Recently nanoscale growth twins were introduced into ultrafine grain coppers, and strengthen behavior was observed very obviously in experiments. ${ }^{29}$ Fivefold deformation twins in nanocrystalline copper were observed in both experiment and MD simulations. ${ }^{30,31}$ This type of nanostructure, which is ubiquitous in the nanoscale materials, has been attractive in controlling the mechanical properties of nanomaterials. 


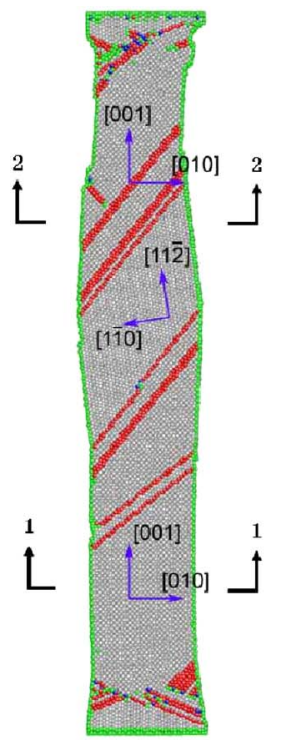

(a)

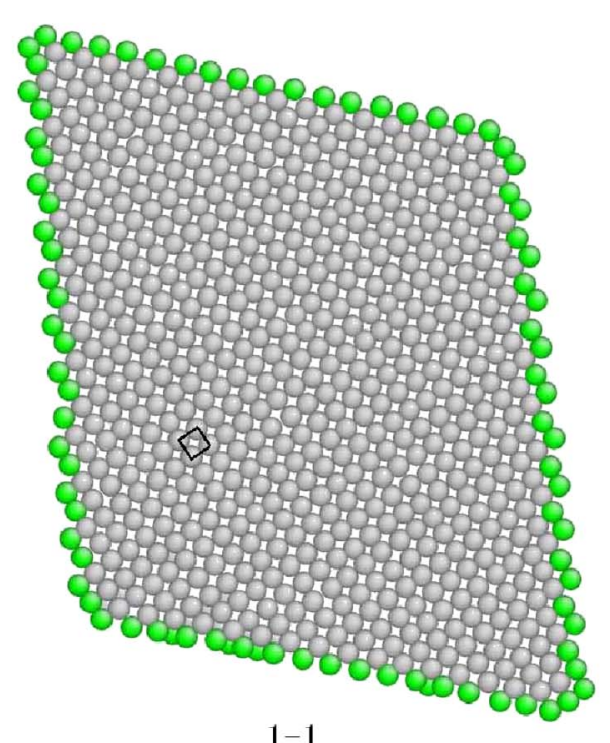

(b)

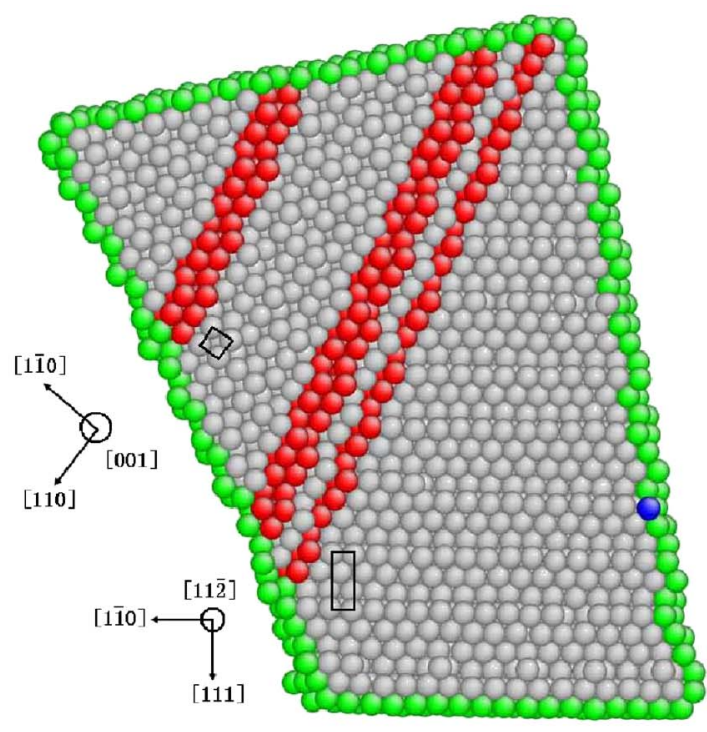

$2-2$

(c)

FIG. 9. (Color online) Second stage of lattice reorientation of the $\langle 110\rangle$ single-crystalline Cu nanowire at a strain of 0.62 . (a) Sectional view along the wire axis and initial [112] of the cross section as Fig. 8(a), different wire axis direction is also shown. (b) Cross-section 1-1 shows the first reoriented $\langle 100\rangle$ lattice configuration with rhombic cross section. (c) Cross-section 2-2 shows the transition region containing both $\langle 100\rangle$ and $\langle 112\rangle$ lattice configurations. Atoms are colored according to CNA.

\section{SUMMARY}

In summary, we have performed atomistic simulations to investigate elastic, inelastic, and failure properties of $\mathrm{Cu}$ nanowires with a fivefold twinned structure. In comparison,

(a)

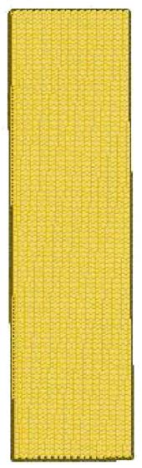

FIG. 10. (Color online) Tensile failure of $\langle 110\rangle$ singlecrystalline $\mathrm{Cu}$ nanowire. (a) Initial equilibrated configuration. (b) Strain at 0.52. (c) Strain just prior to failure. (d) Ultimated fracture strain at 0.86 . we have also performed simulations on the $\langle 110\rangle$ singlecrystalline $\mathrm{Cu}$ wire with two $\{111\}$ side surfaces and two $\{112\}$ surfaces. The yield mechanism for the fivefold twinned $\mathrm{Cu}$ nanowire is via nucleation of $\{111\}\langle 112\rangle$ partial dislocations from surface edge. The simulated results have shown that this twinned nanowire displays higher strength than that

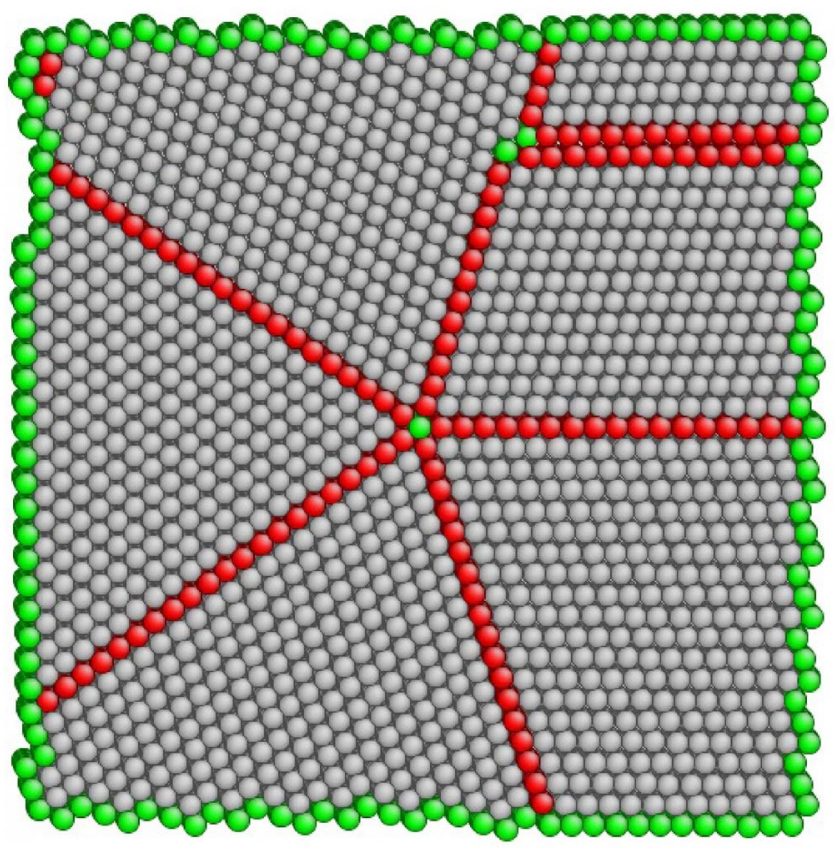

FIG. 11. (Color online) Cross-sectional view of equilibrated configuration of fivefold twinned $\mathrm{Cu}$ nanowire with a stacking fault in it. Atoms are colored according to CNA. 


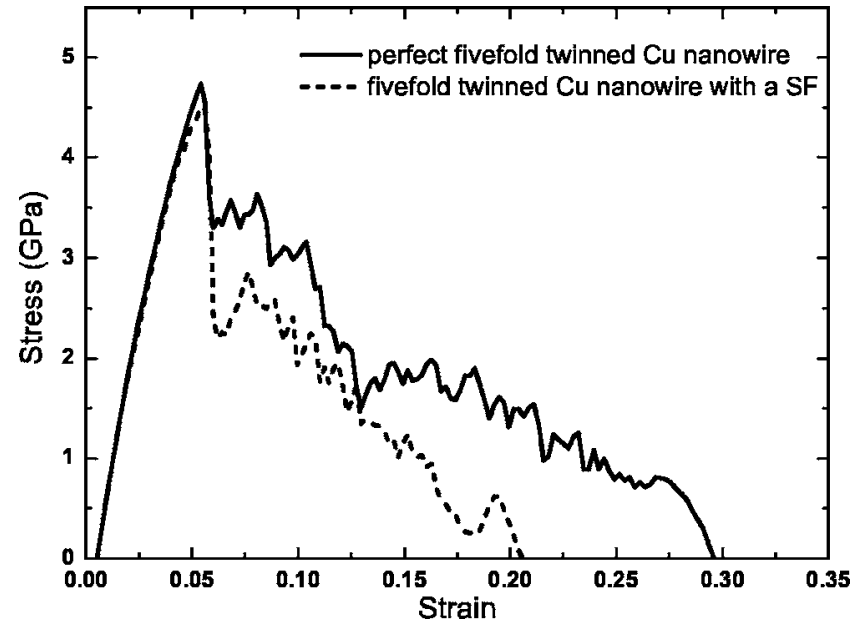

FIG. 12. Tensile stress-strain curves of a perfect fivefold twinned $\mathrm{Cu}$ nanowire and the same nanowire with a preexisting stacking fault in it.

of the single-crystalline fcc $\langle 110\rangle \mathrm{Cu}$ nanowire. The primary reason is that the twin boundaries block dislocation emission and movements and, as a consequence, make wires strengthen, which is in agreement with experimental mea- surements. However, as dislocation movement is limited in the nanowires, brittle failure and low elongation ductility have occurred. In contrast, under the uniaxial tensile loading at room temperature, the $\langle 110\rangle$ wire with $\{111\}$ and $\{112\}$ side surfaces also shows lattice reorientation within the fcc crystalline structure. The deformation stage includes two major steps: (1) $\langle 110\rangle$ wires reorient to $\langle 100\rangle$ wires with $\{100\}$ and $\{112\}$ side surfaces; (2) the newly formed $\langle 100\rangle$ wires reorient to $\langle 112\rangle$ wires with $\{111\}$ side surfaces. Both lattice reorientations occur via the mechanical twinning of fcc structure. In addition, we have investigated the influence of the preexisting defects, such as stacking faults and dislocations on the strength of the novel nanowires.

\section{ACKNOWLEDGMENTS}

The authors gratefully acknowledge financial support from Chinese Academy of Sciences and from National Science Foundation of China through Grants No. 10432050, No. 10428207, and No. 10672163. A.J.C. thanks S. J. Plimpton from Sandia National laboratory for sharing his MD code ParaDyn (Ref. 32) for EAM potential. Computations are carried out at supercomputing centre of Chinese Academy of Science.
*Electronic address: chaoajing@1nm.imech.ac.cn

†Electronic address: Ywei@lnm.imech.ac.cn

${ }^{1}$ E. W. Wong, P. E. Sheehan, and C. M. Lieber, Science 277, 1971 (1997).

${ }^{2}$ A. Hasmy and E. Medina, Phys. Rev. Lett. 88, 096103 (2002).

${ }^{3}$ U. Landman, W. D. Luedtke, B. E. Salisbury, and R. L. Whetten, Phys. Rev. Lett. 77, 1362 (1996).

${ }^{4}$ Y. Kondo and K. Takayanagi, Phys. Rev. Lett. 79, 3455 (1997).

${ }^{5}$ Y. Kondo and K. Takayanagi, Science 289, 606 (2000).

${ }^{6}$ E. Z. da Silva, A. J. R. da Silva, and A. Fazzio, Phys. Rev. Lett. 87, 256102 (2001).

${ }^{7}$ B. Wu, A. Heidelberg, and J. J. Boland, Nat. Mater. 4, 525 (2005).

${ }^{8}$ B. Wu, A. Heidelberg, J. J. Boland, J. E. Sader, X. Sun, and Y. Li, Nano Lett. 6, 468 (2006).

${ }^{9}$ X. M. Sun and Y. D. Li, Adv. Mater. (Weinheim, Ger.) 17, 2626 (2005).

${ }^{10}$ M. S. Daw and M. I. Baskes, Phys. Rev. B 29, 6443 (1984).

${ }^{11}$ S. M. Foiles, M. I. Baskes, and M. S. Daw, Phys. Rev. B 33, 7983 (1986).

${ }^{12}$ K. S. Cheung and S. Yip, J. Appl. Phys. 70, 5688 (1991).

${ }^{13}$ M. Zhou, Proc. R. Soc. London, Ser. A 459, 2347 (2003).

${ }^{14}$ J. Diao, K. Gall, M. L. Dunn, and J. A. Zimmerman, Acta Mater. 54, 643 (2006).

${ }^{15}$ J. D. Honeycutt and H. C. Andersen, J. Phys. Chem. 91, 4950
(1987).

${ }^{16}$ J. Howe, Interfaces in Materials (Wiley, New York, 1997).

${ }^{17}$ S. Nosé, J. Chem. Phys. 81, 511 (1984).

${ }^{18}$ W. G. Hoover, Phys. Rev. A 31, 1695 (1985).

${ }^{19}$ J. Diao, K. Gall, and M. L. Dunn, Nat. Mater. 2, 656 (2003).

${ }^{20}$ J. Diao, K. Gall, and M. L. Dunn, Nano Lett. 4, 1863 (2004).

${ }^{21}$ K. Gall, J. Diao, and M. L. Dunn, Nano Lett. 4, 2431 (2004).

${ }^{22}$ W. Liang and M. Zhou, J. Eng. Mater. Technol. 127, 423 (2005).

${ }^{23}$ W. Liang, M. Zhou, and F. Ke, Nano Lett. 5, 2039 (2005).

${ }^{24}$ W. Liang and M. Zhou, Phys. Rev. B 73, 115409 (2006).

${ }^{25}$ H. S. Park and J. A. Zimmerman, Phys. Rev. B 72, 054106 (2005).

${ }^{26}$ H. S. Park, K. Gall, and J. A. Zimmerman, Phys. Rev. Lett. 95, 255504 (2005)

${ }^{27}$ Y. Mishin, M. J. Mehl, D. A. Papaconstantopoulos, A. F. Voter, and J. D. Kress, Phys. Rev. B 63, 224106 (2001).

${ }^{28}$ V. Rodrigues, T. Fuhrer, and D. Ugarte, Phys. Rev. Lett. 85, 4124 (2000).

${ }^{29}$ L. Lu, Y. Shen, X. Chen, L. Qian, and K. Lu, Science 304, 422 (2004).

${ }^{30}$ X. Z. Liao, Y. H. Zhao, S. G. Srinivasan, Y. T. Zhu, R. Z. Valiev, and D. V. Gunderov, Appl. Phys. Lett. 84, 592 (2004).

${ }^{31}$ A. J. Cao and Y. G. Wei, Appl. Phys. Lett. 89, 041919 (2006).

${ }^{32}$ S. J. Plimpton, J. Comput. Phys. 117, 1 (1995). 